\title{
Precision study of the $S U(3)$ topological susceptibility in the continuum
}

\author{
Stephan Dürr ${ }^{a}$, Zoltan Fodor ${ }^{b c d}$, Christian Hölbling $^{b e}$, Thorsten Kurth ${ }^{* b}$ \\ ${ }^{a}$ Institut für theoretische Physik, Universität Bern \\ Sidlerstr. 5, CH-3012 Bern, Switzerland \\ ${ }^{b}$ Fachbereich C - Mathematik und Naturwissenschaften, Bergische Universität Wuppertal \\ Gaussstr. 20, D-42119 Wuppertal, Germany \\ ${ }^{c}$ Physics Department, Eötvös University \\ Pázmány 1, H-1117 Budapest, Hungary \\ ${ }^{d}$ University of California at San Diego \\ 9500 Gilman Drive, La Jolla, CA 92093-0319 USA \\ ${ }^{e}$ Centre de Physique Théorique ${ }^{\dagger}$ \\ Marseille, France
}

We determine the topological susceptibility in the $S U(3)$ pure gauge theory by performing a series of high-statistics studies. We calculate the combined continuum and infinite volume limit and obtain $\chi_{\text {top }} r_{0}^{4}=0.0524(7)(6)$, which translates into $\chi_{\text {top }}^{1 / 4}=193(1)(8) \mathrm{MeV}$, where the second error is exclusively caused by the intrinsic scale setting ambiguity in $S U(3)$ Yang-Mills-theory.

The XXV International Symposium on Lattice Field Theory

July 30 - August 42007

Regensburg, Germany

\footnotetext{
* Speaker.

${ }^{\dagger}$ UMR 6207 du CNRS et des universités d'Aix-Marseille I, II et du Sud Toulon-Var, affiliée à la FRUMAM
} 


\section{Introduction}

In our study we considered the quenched topological susceptibility $\chi_{\text {top }}^{Y M}$ which is linked via the famous Witten-Veneziano formula [1,2]

$$
\chi_{\mathrm{top}}^{Y M}=\frac{F^{2}}{2 N_{f}}\left(M_{\eta^{\prime}}^{2}+M_{\eta}^{2}-2 M_{K}^{2}\right)
$$

to the excess of the $\eta^{\prime}$ mass over the pseudoscalar octet masses, where the proportionality factor contains the pseudoscalar decay constant $F=86.2(5) \mathrm{MeV}$ in the chiral limit ${ }^{1}$. This relation holds at leading order in the large $N_{c}$ expansion and connects full QCD with the corresponding theory at zero virtuality. We study with unprecedented precision the quenched topological susceptibility for the case $N_{c}=3$. We start by defining a topological charge for the lattice and explain how it is possible to extract the topological susceptibility in the continuum. The next sections contain details of our lattice simulations as well as the analysis of our results. We take the continuum as well as the infinite volume limit and obtain a value for $\chi_{\text {top }}^{Y M}$ in terms of the Sommer radius $r_{0}$ [3]. To convert this value into physical units, it is necessary to set the scale and discuss it's intrinsic ambiguity.

\section{Topological charge definition}

In the continuum the topological charge $q$ of a given gauge background is given by

$$
q=\frac{1}{16 \pi^{2}} \int \mathrm{d}^{4} x \operatorname{Tr}\left[F_{\mu v}(x) \tilde{F}^{\mu v}(x)\right]
$$

where $\tilde{F}_{\mu \nu}=\varepsilon_{\mu v \alpha \beta} F^{\alpha \beta} / 2$ and $F_{\mu \nu}=F_{\mu \nu}^{a} \lambda^{a} / 2$ is the field strength tensor. On a toroidal spacetime geometry (2.1) is integer and linked to the index of the Dirac operator $D$ via the Atiyah-Singer theorem [4]

$$
q=n_{-}-n_{+},
$$

where $n_{ \pm}$denotes the number of zero modes of $D$ with positive or negative chirality. The lattice itself is no manifold and therefore there is no unique definition of the topological charge for its geometry $T^{4}$. The simplest choice, which we will call the "naive charge", is of course the straightforward discretization of (2.1):

$$
q_{\text {nai }} \doteq \frac{1}{16 \pi^{2}} \sum_{x \in T^{4}} \operatorname{Tr}\left[F_{\mu v}(x) \tilde{F}^{\mu v}(x)\right],
$$

where one also has to apply a suitable discretization of $F_{\mu \nu}$ which has the correct continuum limit. This definition in general does not yield integer values. It is possible to discretize the fermionic definition (2.2) in a way, that it will always give integer values (cf. $[15,16])$, but this is computationally very expensive.

In the continuum the topological susceptibility $\chi_{\text {top }}$ is defined as

$$
\chi_{\mathrm{top}} \doteq \lim _{V \rightarrow \infty} \frac{\left\langle q^{2}\right\rangle}{V},
$$

\footnotetext{
${ }^{1}$ We use the Bern normalization where $F_{\pi}^{\text {phys }}=92.4(3) \mathrm{MeV}$.
} 
what shows that a finite volume is mandatory for its definition. Contributions which grow less than linearly in $V$ will create finite volume effects in $\chi_{\text {top }}$. It is obvious that the lattice definition of $\chi_{\text {top }}$ is very sensitive to the definition of the topological charge $q$. The traditional approach is to calculate $\left\langle q_{\text {nai }}^{2}\right\rangle / V$ and then to apply a multiplicative as well as an additive renormalization [5-7]. Using the integer-valued fermionic charge $q_{\mathrm{fer}}$ instead renders the additive renormalization unnecessary. On the other hand, as already mentioned, such a definition is very expensive and therefore not suitable for a high precision lattice study of the topological susceptibility. In this project we followed a different approach, namely by renormalizing the naive charge and casting it to integer values. This is done in the following way: we used the standard "clover-leaf" definition for $F_{\mu \nu}$ (which uses the average of the antihermitean part of the plaquette $P_{\mu v}$ at $\left.x, x-\hat{\mu}, x-\hat{v}, x-\hat{\mu}-\hat{v}\right)$, based on (p-)HYP smeared [8] gauge links. Plugging this into 2.3 we obtain the bare charge $q_{\text {nai }}$ which is a real number. One of our $q_{\text {nai }}$ distributions ( $\beta=6.0,12^{4}, 3$ HYP steps) is displayed on the left panel of Fig.1. Due to CP symmetry, only a multiplicative renormalization applies and we opt for a non-perturbatively defined $Z$-factor. We make use of the fact, that the overall distribution $q_{\text {nai }}$ tends to cluster near integer values (cf. 1). We obtain $Z$ by minimizing

$$
\chi^{2}=\sum_{U}\left(Z q_{\text {nai }}[U]-\operatorname{round}\left(Z q_{\text {nai }}[U]\right)\right)^{2}
$$

with the constraint $Z>1^{2}$ and use it to define the renormalized field-theoretic charge

$$
q_{\mathrm{ren}}[U] \doteq \operatorname{round}\left(Z q_{\mathrm{nai}}[U]\right),
$$

where round means rounding to the nearest integer. With this charge definition, we set

$$
\chi_{\text {top,lat }}(a, V) \doteq \frac{\left\langle q_{\text {nai }}^{2}\right\rangle}{V}
$$

and take the combined continuum and infinte volume limit in order to obtain the topological charge in the continuum. Indeed, the rounding procedure introduces a global element and therefore our definition of the topological charge cannot be rewritten as an integral over a local density. Accordingly, there is no conflict with the result by Stamatescu and Seiler that $q(x)$ in general mixes with the identity and $\int \mathrm{d}^{4} x q(x) q(0)$ has a contact term [14]. Our topological susceptibility vanishes in the zero-charge sector and an additive renormalization of $\chi_{\text {top,lat }}$ is therefore absent.

\section{Lattice simulations and results}

We performed two series of runs: one for doing the continuum- and the second for doing the infinite volume extrapolation. We use the MILC code [9] to produce the $S U(3)$ gauge ensembles a different $\beta$-values and lattice sizes. In the first run, the lattice size and coupling $\beta$ was varied in order to keep the physical volume fixed at $V=\left(2.2394 r_{0}\right)^{4}$. The dependence of $a$ on $\beta$ and $r_{0}$ is given by the Necco Sommer data [10], where we applied a different fit model [13]. We aimed at $10^{5}$ measurements per run, where we adapted the separation between the measurements when simulating at larger $\beta$ in order to keep the autocorrelation time approximately constant. As a first

\footnotetext{
${ }^{2}$ This is to avoid the global minimum at $Z=0$ [11]. Perturbative studies also show that $Z=1+$ const $/ \beta>1$ [12].
} 

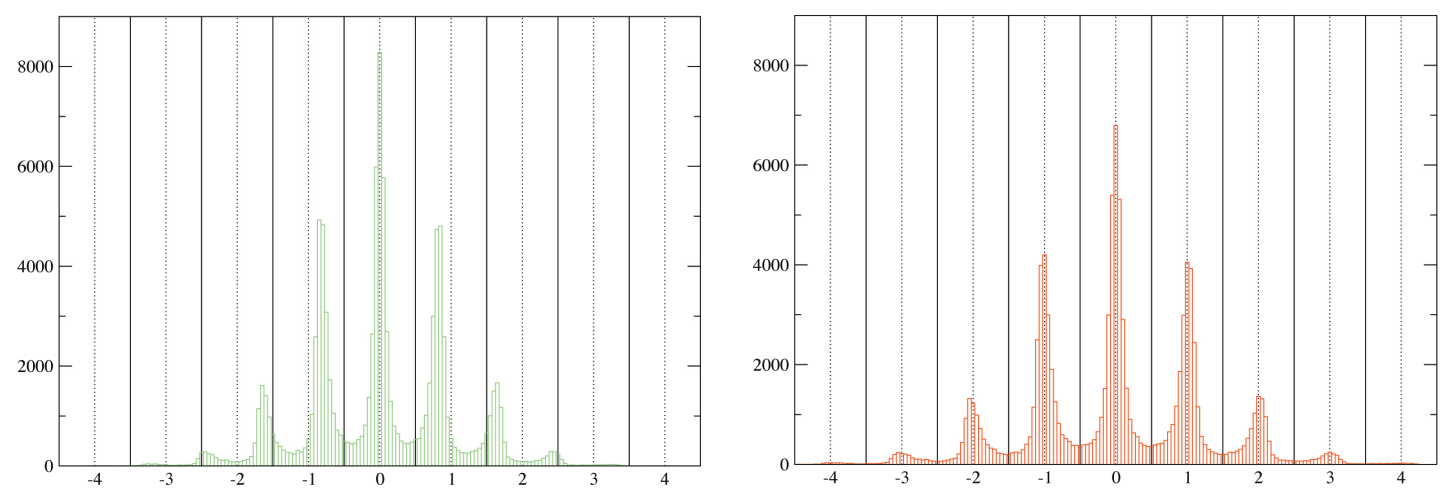

Figure 1: Histogram of the topological charge $q_{\text {nai }}$ with 3 HYP steps for the $12^{4}$ lattices at $\beta=6.0$, before and after rescaling with the renormalization factors defined in (2.5).
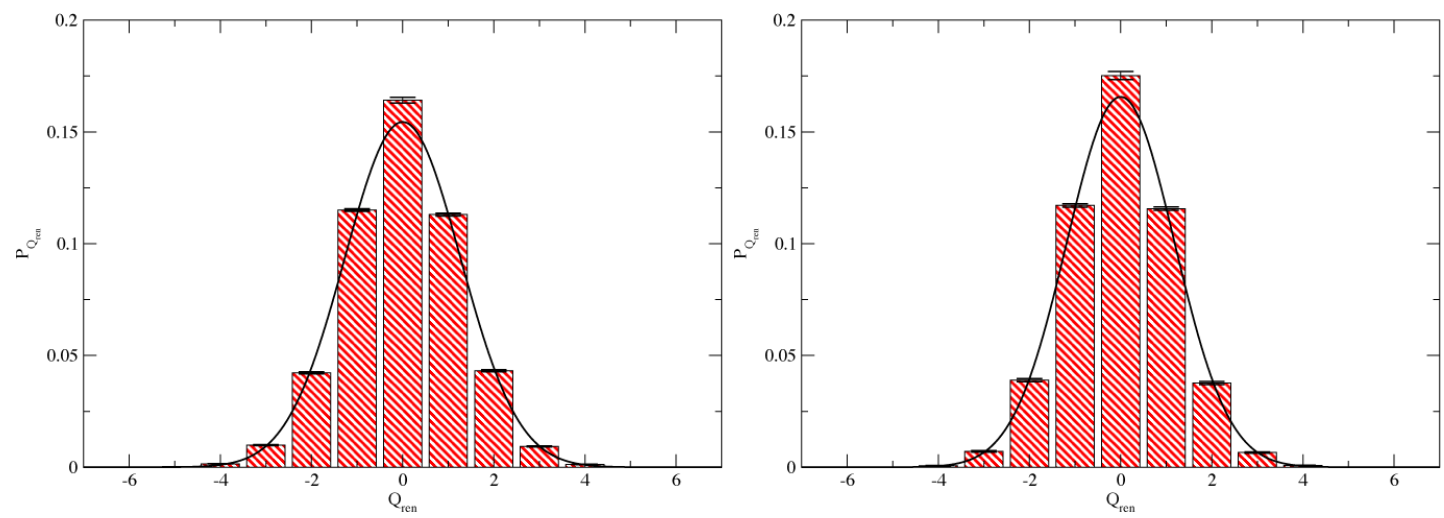

Figure 2: Histogram of the renormalized topological charge $q_{\text {ren }}$ for the $\left(6.0,12^{4}\right)\left[\right.$ left] and $\left(6.3344,20^{4}\right)$ lattices [right], where the Gaussian fit excludes the $q=0$ sector. The excess gives rise to the kurtosis $\left\langle q^{4}\right\rangle /\left\langle q^{2}\right\rangle^{2}-3$ (cf. Fig.4(a)).

check, we calculated $|\langle q\rangle|$ and found it consistent with zero on all lattices. The observable of interest $\left\langle q^{2}\right\rangle$ is measured with $1 \%$ accuracy or better throughout. In addition we checked whether using two or four steps of HYP smearing changes our results significantly, but this is not the case. The histogram of the renormalized charge $q_{\mathrm{ren}}$ for $(\beta=6.0, L / a=12)$ is displayed in Fig.2. It turns out that the relative weight of the topologically non-trivial sectors is almost consistent with a Gaussian, while the $q=0$ sector shows a clear excess. This leads us to the definition of a new estimator $\left\langle q_{\mathrm{ren}}^{2}\right\rangle_{q \neq 0}$, which should be unsensitive to this excess. It is computed by symmetrizing the charge histogram and fitting it to a half-Gaussian, where the zeroth bin is neglected. The width of this Gaussian is then denoted by $\left\langle q_{\mathrm{ren}}^{2}\right\rangle_{q \neq 0}$. The continuum limit of both, the naive and the new estimator, is shown in Fig.3(a).

By applying a linear fit ${ }^{3}$, we obtain the significantly different continuum limits $\left\langle q^{2}\right\rangle\left(r_{0} / L\right)^{4}=$ $0.05092(71)$ and $\left\langle q^{2}\right\rangle_{q \neq 0}\left(r_{0} / L\right)^{4}=0.05205(71)$ respectively. We assume that this difference is caused by a finite volume effect, which brings us to the discussion of the second series of runs.

Finite volume effects are attributed to infrared physics and we assume that our IR and UV modes

\footnotetext{
${ }^{3}$ and dropping the point at the coarsest lattice $(\beta=5.898, L / a=10)$
} 


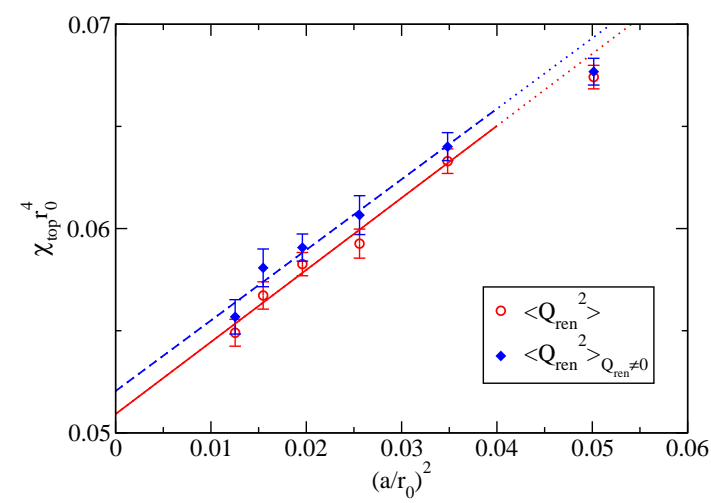

(a) Continuum extrapolation in the fixed physical volume $V=$ $\left(2.239 r_{0}^{4}\right)^{4}$.

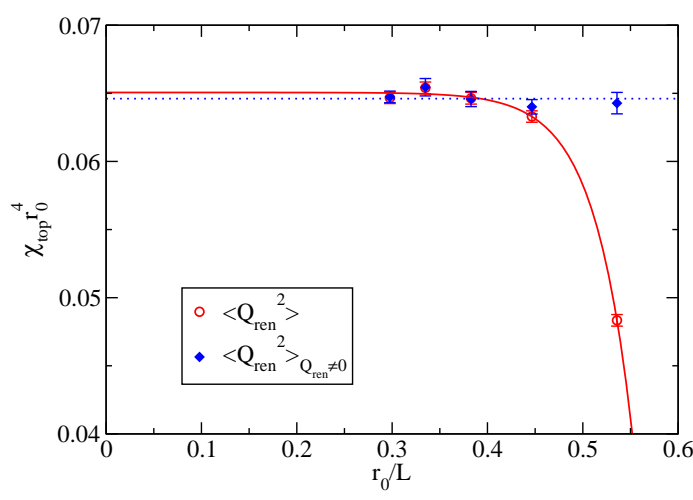

(b) Infinite volume extrapolation vs. $r_{0} / L$.

Figure 3: Different extrapolations of $\chi_{\mathrm{top}} r_{0}^{4}$.

decouple. Therefore ist is sufficient to restrict ourselves to a single coupling $\beta=6.0$. Usually, finite volume effects are caused by massive states travelling around the box. Because we are simulating in a world without quarks, these states have to be glueball states with a mass $M_{G}$. These states imply corrections to the partition function of the following form

$$
Z(L)=Z(\infty)\left(1+\text { const } e^{-M_{G} L}+\ldots\right)
$$

If we assume these exponential finite-volume effects, the data for the naive estimator may be fitted all the way out to $L / a=10$ and suggests a correction $\Delta \chi_{\text {top,red }}=0.00175$. The dataset coming from the second estimator is unaffected by finite volume corrections (cf. Fig.3(b)), so the corresponding continuum limit does not need any correction. Hence we obtain $\left\langle q^{2}\right\rangle\left(r_{0} / L\right)^{4}=0.05267(71)$ and $\left\langle q^{2}\right\rangle_{q \neq 0}\left(r_{0} / L\right)^{4}=0.05205(71)$. Taking the mean and the full difference as our systematic error, we obtain for the topological susceptibility in the continuum

$$
\chi_{\text {top }} r_{0}^{4}=0.05236(71)(62)
$$

where the first error is statistical and the second systematical.

We also verified directly that the excess in the zeroth bin of the populations in Fig.2 is a pure finite volume effect. Therefore we measured the kurtosis $\left\langle q^{4}\right\rangle /\left\langle q^{2}\right\rangle^{2}-3$, a quantity which measures the deviation of a given distribution from a Gaussian. It turns out that this quantity does not vanish in the continuum extrapolation series of runs (cf. Fig.4(a)), as anticipated when having a closer look at Fig.2. But when having a look at the infinite volume behaviour of this quantity, we find a vanishing kurtosis (cf. Fig.4(b)).

We also measured the reduced moment ratio $\left\langle q^{4}\right\rangle /\left\langle q^{2}\right\rangle-3\left\langle q^{2}\right\rangle$ and performed the infinite volume limit. Here, we are consistent with the value $0.276(84)$ given in [17], but we cannot rule out the possibility that the infinite volume limit of this quantity might actually vanish. 


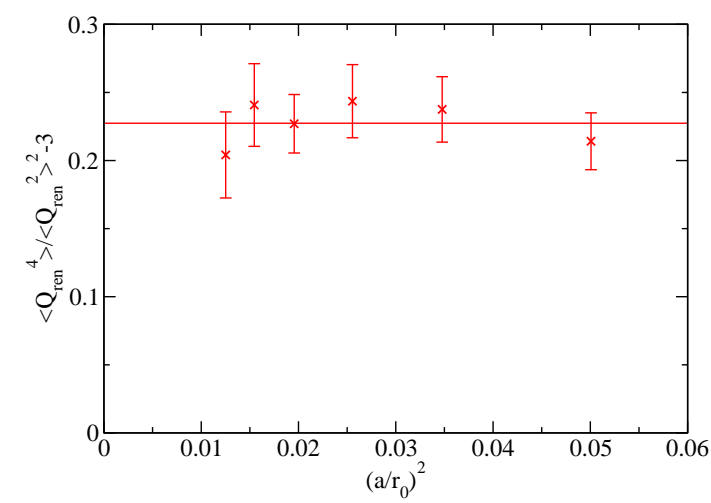

(a) Continuum extrapolation in the fixed physical volume $V=$ $\left(2.239 r_{0}^{4}\right)^{4}$.

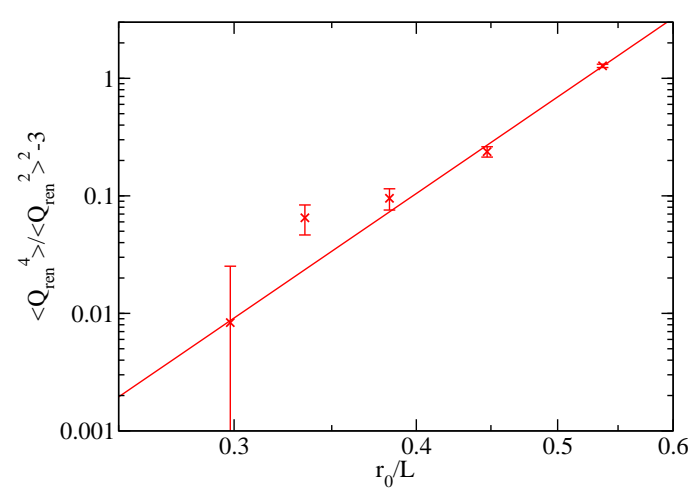

(b) Infinite volume extrapolation in log-log representation.

Figure 4: Different extrapolations of $\left\langle q^{4}\right\rangle /\left\langle q^{2}\right\rangle^{2}-3$.

\section{Conversion to physical units}

Finally we convert our unambiguous result for the topological susceptibility in the combined continuum and infinite volume limit in units of $r_{0}^{-1}$ into physical units. Therefore we have to assume a value (in $\mathrm{fm}$ ) for $r_{0}$. In full QCD, $r_{0}$ is a well defined quantity and can be determined by measuring $a M_{p}, a M_{\pi}$ and $a M_{K}$ in $2+1$ flavour simulations in which their ratios are adjusted to the corresponding experimental value. By considering $r_{0} M_{p}$, one then would obtain the correct physical value. Of course, one can use the original value of $r_{0}=0.5 \mathrm{fm}$ also in YM theory, but there are different estimates coming from Kaon decay measurements $\left(r_{0}=0.512(12) \mathrm{fm}\right)$ or recent studies which give $r_{0}=0.467(6) \mathrm{fm}$. To reflect this ambiguity, we choose $r_{0}=0.49 \mathrm{fm}$ and add a $4 \%$ error. With this choice, we find for the topological susceptibility in physical units

$$
\chi_{\text {top }}^{1 / 4}=193(1)(8) \mathrm{MeV},
$$

where the first error bar contains all statistical and systematic uncertainties of our calculation, and the second one reflects the scale setting ambiguity in a theory which is different from full QCD.

\section{References}

[1] E. Witten, Current Algebra Theorems for the U(1) Goldstone Boson, Nucl. Phys. B156 (1979) 269

[2] G. Veneziano, U(1) Without Instantons, Nucl. Phys. B159 (1979) 213

[3] R. Sommer, A New way to set the energy scale in lattice gauge theories and its applications to the static force and alpha-s in SU(2) Yang-Mills theory, Nucl. Phys. B411 (1993) 839, hep-lat/9310022

[4] M.F. Atiyah and I.M. Singer, The Index of elliptic operators. 1, Annals Math. 87 (1968) 484

[5] M. Campostrini, A. Di Giacomo, H. Panagopoulos and E. Vicari, Topological Charge, Renormalization And Cooling On The Lattice, Nucl. Phys. B329 (1990) 683 
[6] B. Alles, M. D'Elia, A. Di Giacomo and R. Kirchner, Topology in SU(2) Yang-Mills theory, Phys. Rev. D58 (1998) 114506

[7] A. Di Giacomo and E. Vicari, Renormalization and topological susceptibility on the lattice, Phys. Lett. B275 (1992) 429

[8] A. Hasenfratz and F. Knechtli, Flavor symmetry and the static potential with hypercubic blocking, Phys. Rev. D64 (2001) 034504, hep-lat/ 0103029

[9] http://www.physics.utah.edu/ detar/milc

[10] S. Necco and R. Sommer, The $N(f)=0$ heavy quark potential from short to intermediate distances, Nucl. Phys. B622 (2001) 328, hep-lat / 0108008

[11] S. Dürr, Gauge action improvement and smearing, Comput. Phys. Commun. 172 (2004) 163, hep-lat/0409141

[12] C. Christou, A. Di Giacomo, H. Panagopoulos and E. Vicari, Improved lattice operators: The Case of the topological charge density, Phys. Rev. D53 (1995) 2619, hep-lat/ 9510023

[13] S. Dürr, Z. Fodor, C. Hölbling and T. Kurth, Precision study of the SU(3) topological susceptibility in the continuum, JHEP 0704 (2006) 055, hep-lat / 0612021

[14] E. Seiler and I.O. Stamatescu, MPI-PAE/PTH 10/87 (unpublished)

[15] P. Hasenfratz, V. Laliena and F. Niedermayer, The Index theorem in QCD with a finite cutoff, Phys. Lett. B427 (1998) 125, hep-lat/9801021

[16] M. Lüscher, Exact chiral symmetry on the lattice and the Ginsparg-Wilson relation, Phys. Lett. B428 (1998) 342, hep-lat/9802011

[17] L. Del Debbio, H. Panagopoulos and E. Vicari, theta dependence of SU(N) gauge theories, JHEP 0208 (2002) 044, hep-th/ 0204125 